\title{
Special issue on NETYS'17 selected papers
}

\author{
Yahya Benkaouz ${ }^{1} \cdot$ Mohammed Erradi $^{2}$
}

Published online: 24 July 2019

๑) Springer-Verlag GmbH Austria, part of Springer Nature 2019

This special issue comprises a selection of best papers presented at the International Conference on Networked Systems: NETYS'17. The conference gathers researchers and practitioners to share new ideas mainly related to distributed and networked systems. The selected papers tackle important problems such as Security policies formalization and verification; Customer behavior analysis in telecommunication networks; Exploration of relaxation methods of shared data structure and finally, Study of dynamic clustering problem.

In the paper entitled "Policy Expressions and the Bottom-Up Design of Computing Policies", the authors suggest a generalization expression of access control policies that might be used to support bottom-up methods to design policies. The suggested policy expression facilitates the verification of several logical properties. The authors of the paper "On Understanding Price-QoS War for Competitive Market and Confused Consumers" makes use of non-cooperative game theory to model and study the impact of customer behavior and their bounded rationality in the telecommunication networks.

In the paper "Anomalies and Similarities among Consensus Numbers of VariouslyRelaxed Queues", the authors study the tradeoff on the computational power of FIFO queue due to various relaxations. Finally, the authors of "Competitive Clustering of Stochastic Communication Patterns on a Ring" discuss the problem of assigning nodes to clusters in order to minimize cross-cluster communication costs. They suggest an algorithm that decides on the fly whether to migrate nodes, or stay in the same configuration.

Acknowledgements We would like to thank all the authors of NETYS 2017 as well as the reviewers of the selected papers of this special issue who thoroughly evaluated their quality through a rigorous process.

Publisher's Note Springer Nature remains neutral with regard to jurisdictional claims in published maps and institutional affiliations.

\footnotetext{
$\triangle$ Yahya Benkaouz

y.benkaouz@um5s.net.ma

Mohammed Erradi

mohamed.erradi@gmail.com

1 FSR, Mohammed V University in Rabat, Rabat, Morocco

2 ENSIAS, Mohammed V University in Rabat, Rabat, Morocco
} 